\title{
Household food insecurity is associated with low dietary diversity among pregnant and lactating women in rural Malawi
}

\author{
Yunhee Kang ${ }^{1, *}$, Kristen M Hurley ${ }^{1}$, Julie Ruel-Bergeron ${ }^{1}$, Assumpta Bou Monclus ${ }^{1}$, \\ Rachel Oemcke ${ }^{1}$, Lee Shu Fune Wu ${ }^{1}$, Maithilee Mitra ${ }^{1}$, John Phuka ${ }^{2}$, Rolf Klemm ${ }^{1,3}$, \\ Keith P West $\mathrm{Jr}^{1}$ and Parul Christian ${ }^{1,4}$ \\ ${ }^{1}$ Center for Human Nutrition, Department of International Health, Johns Hopkins Bloomberg School of Public Health, \\ 615 N. Wolfe Street, Baltimore, MD 21205, USA: ${ }^{2}$ College of Medicine, University of Malawi, Blantyre, Malawi: \\ ${ }^{3}$ Nutrition, Helen Keller International, New York, NY, USA: ${ }^{4}$ Bill \& Melinda Gates Foundation, Seattle, WA, USA
}

Submitted 19 March 2018: Final revision received 31 August 2018: Accepted 13 September 2018: First published online 31 October 2018

\begin{abstract}
Objective: To examine the association between household food insecurity and dietary diversity in the past $24 \mathrm{~h}$ (dietary diversity score (DDS, range: 0-9); minimum dietary diversity (MDD, consumption of three or more food groups); consumption of nine separate food groups) among pregnant and lactating women in rural Malawi.

Design: Cross-sectional study.

Setting: Two rural districts in Central Malawi.

Subjects: Pregnant ( $n$ 589) and lactating ( $n$ 641) women.

Results: Of surveyed pregnant and lactating women, 66.7 and $68.6 \%$, respectively, experienced moderate or severe food insecurity and only 32.4 and $28.1 \%$, respectively, met MDD. Compared with food-secure pregnant women, those who reported severe food insecurity had a 0.36 lower DDS $(P<0.05)$ and more than threefold higher risk (OR; 95\% CI) of not consuming meat/fish (3.19; CI 1.68, 6.03). The risk of not consuming eggs $(3.77 ; 1.04,13.7)$ was higher among moderately food-insecure pregnant women. Compared with food-secure lactating women, those who reported mild, moderate and severe food insecurity showed a $0.36,0.44$ and 0.62 lower DDS, respectively (all $P<0.05$ ). The risk of not achieving MDD was higher among moderately $(1.95 ; 1.06,3.59)$ and severely $(2 \cdot 82 ; 1.53$, $5 \cdot 22$ ) food-insecure lactating women. The risk of not consuming meat/fish and eggs increased in a dose-response manner among lactating women experiencing mild $(1.75 ; 1.01,3.03$ and $2.81 ; 1.09,7.25)$, moderate $(2.66 ; 1.47,4.82$ and 3.75 ; $1 \cdot 40,10 \cdot 0)$ and severe $(5 \cdot 33 ; 2 \cdot 63,10 \cdot 8$ and $3 \cdot 47 ; 1 \cdot 19,10 \cdot 1)$ food insecurity. Conclusions: Addressing food insecurity during and after pregnancy needs to be considered when designing nutrition programmes aiming to increase dietary diversity in rural Malawi.
\end{abstract}

Appropriate nutrition during pregnancy and lactation is essential, given increased nutritional requirements during these periods, to promote both maternal and newborn growth and well-being. In resource-poor settings, pregnant women are often exposed to a low dietary diversity (or quality), which contributes to inadequate macronutrient and micronutrient intakes ${ }^{(1-4)}$. Undernutrition during pregnancy may lead to increased risks of obstetric and neonatal complications including preterm birth, intra-uterine growth retardation and low birth weight, which further increase the risk of adverse health consequences among women and children alike ${ }^{(5)}$. In lactating women, a poor diet may affect the nutrient quality of breast milk ${ }^{(6-8)}$.

UNICEF's conceptual framework of malnutrition describes that household food insecurity leads to inadequate dietary intake ${ }^{(9)}$. A few studies have reported associations between poor dietary diversity and food insecurity among reproductive-aged women in resource-poor settings ${ }^{(10-13)}$. However, the association has not been studied extensively among pregnant and lactating women (PLW), except for Bangladeshi pregnant mothers ${ }^{(14-16)}$.

According to the Malawi Demographic and Health Survey $2015-16,45 \cdot 1 \%$ of pregnant women and $29 \cdot 3 \%$ of 
lactating mothers are anaemic ${ }^{(17)}$. This condition may be partially due to insufficient dietary intake of $\mathrm{Fe}^{(18,19)}$. Since the 1990s, the Malawian government has provided free or subsidized input programmes, focusing primarily on maize production among smallholder farmers, prioritizing the poor ${ }^{(20)}$. According to a study using data from the Malawi Integrated Household Survey (IHS) 2004/2005 and IHS 2010/2011, per capita food and energy consumption and dietary diversity increased nationwide ${ }^{(21)}$. However, consumption of some key micronutrients declined in rural areas, including vitamin $\mathrm{A}$, folate, $\mathrm{Fe}$ and $\mathrm{Zn}^{(21)}$.

In the present study we sought to understand how household food insecurity is associated with dietary diversity among pregnant women and lactating women in rural Malawi. Specifically, we examined the association between severity of household food insecurity and PLW's dietary diversity score (DDS), achievement of minimum dietary diversity (MDD, defined as consumption of three or more food groups in the $24 \mathrm{~h}$ preceding the survey) and consumption of nine food groups.

\section{Methods}

\section{Study setting and population}

The present study was conducted in two rural, neighbouring districts in Central Malawi. The main ethnic group in this area of Malawi is Chewa. Most households practise subsistence farming and are dependent on rain-fed agricultural practices. The main staple crops are maize, sweet potatoes and pulses. Predominant cash crops grown are cotton, tobacco and groundnuts ${ }^{(22)}$. Almost $40 \%$ of households experience some type of food insecurity ${ }^{(23)}$.

The present study used the baseline survey data of a large-scale impact evaluation of a stunting prevention programme, based on a cross-sectional pre-post comparison design with one programme and one comparison district. The stunting prevention programme, led by the Government of Malawi and implemented with technical support from the World Food Programme and World Vision Malawi, targeted children 6 to 23 months of age. The programme district was selected based on a high burden of stunting, government policy and partnership for programme scaling up in future ${ }^{(24)}$. In the comparison district, routine government health service was available. PLW with children less than 6 months of age, and children aged 6 to 23 months in the districts, were to be assessed under the programme impact evaluation.

\section{Data collection}

The baseline survey was conducted from January to March 2014. A total of 1200 children aged 6 to 23 months and 600 PLW was planned per district for the baseline survey. Assuming $6 \%$ of the total population is children 6 to 23 months of age, the required number of villages to be sampled to reach the sample size was estimated at 108 per district (150-600 households per village). In the selected 216 villages, a total of 12529 households were screened, out of which 2307 households with pregnant women and lactating mothers were identified. Using a 2/3 sampling ratio, 1296 households with PLW were visited. Pregnancy was assessed verbally during household screening procedures and at the time of the survey. Lactating women with a child less than 6 months of age were identified via maternal report and verified by the child's date of birth recorded on the health passport/card.

The collected sample size ( $\sim 00$ PLW per district) enabled an $8 \%$ detectable difference in the proportion of women who achieved MDD (defined as consuming at least three food groups out of nine) with $80 \%$ power and $\alpha=0.05$ from baseline of the intervention to endline.

Interviewers were trained to administer structured household questionnaires using standardized field procedures. Pilot testing was conducted prior to the baseline survey. All questionnaires were translated in the local language, Chichewa. Four teams conducted data collection; each comprised one supervisor, three enumerators, one nurse and one vehicle driver. At the completion of data collection, all forms were transported to a data management centre in Zomba, Malawi. Data were double entered into the Census and Survey Processing System (CSPro) version 3.2 (US Census Bureau, 2007). Data entry errors were identified, verified and corrected against corresponding paper forms.

\section{Measures}

Independent variables

Household food insecurity status was measured using a nine-item Household Food Insecurity Access Scale (HFIAS) module that assesses the frequency with which certain aspects of food insecurity are experienced by the household $^{(25)}$. Response options for each item include rarely, sometimes and often (see online supplementary material, Supplemental Table 1). The validity of the HFIAS has been demonstrated in several countries including Tanzania $^{(26)}$, Ethiopia ${ }^{(27)}$ and Burundi ${ }^{(28)}$. This scale reflects three universal domains of household-level food insecurity: (i) anxiety about food supply; (ii) inadequate food quality, meaning the lack of food diversity and decreased availability and access to preferred foods; and (iii) insufficient quantity of food, reflected in lower consumption, and the physical consequences of insufficiency $^{(25)}$. Following the standard categorization of HFIAS, the responses were categorized as four levels of household food insecurity: food secure and mildly, moderately and severely food insecure.

\section{Dependent variables}

Our dietary assessment questionnaire was based on the FAO guidelines for measuring household and individual 
dietary diversity ${ }^{(29)}$. The questionnaire included thirteen food items that were surveyed and aggregated into the nine following food groups: (i) grains/roots; (ii) dark green leafy vegetables (DGLV); (iii) vitamin A-rich vegetables or fruits (VAFV) and red palm oil; (iv) other fruits and vegetables (OFV); (v) organ meat; (vi) meat/poultry/fish/ insects, (vii) eggs; (viii) beans/lentils/nuts/seeds; and (ix) animal milk and dairy foods. Using this questionnaire, a DDS was computed based on the consumption of foods from nine food groups in the previous $24 \mathrm{~h}$. In addition, MDD, which was defined as consumption of three or more food groups out of nine, and the likelihood of consuming foods from nine individual food groups were computed. The threshold of three food groups used for the calculation of MDD was based on the categorization of DDS into 50th upper or lower percentiles. The 50th upper percentile consisted of 3 or more points out of 9 , which was used to set the minimum level of dietary diversity of this study group.

\section{Potential confounders}

Household- and individual-level characteristics were assessed and explored as potential confounding variables. A priori variable selection was based on theory and previous literature and included demographic and socioeconomic variables such as household water sources and sanitation facilities, pregnancy history, education level, occupational status, and health and nutrition service utilization. Household wealth quintiles were created using principal component analysis ${ }^{(30)}$, using twenty-three variables related to ownership of: (i) household assets (number of rooms, electricity, radio, television, bicycle, mobile phones, tape and/or CD player, beds, sofas, tables and chairs, pounding mortar/pestle); (ii) land availability (land ownership, land for rent, land for producing foods, land for producing foods for sale); (iii) any livestock ownership; (iv) years of maternal education; and (v) use of improved toilet facilities. Assets that were surveyed but too infrequent $(<1 \%)$ were excluded from the wealth index construction.

\section{Statistical analysis}

Exploratory data analyses were conducted. Each variable was presented as mean and sD for continuous variables or $n$ and \% for binary/categorical variables. To examine the association between food security and dietary diversity, univariate and multivariable linear or logistic regression models for continuous and binary outcomes, respectively, were conducted. Analyses were conducted separately for pregnant women and lactating women. Potential confounding variables such as district location, household wealth quintiles, drinking-water sources, household size, maternal age, maternal literacy and years of education, maternal occupation, marital status and mid-upper arm circumference were included in multivariable regression models, accounting for village-level clustering. $P<0.05$ was considered statistically significant. All data were analysed using the statistical software package Stata version 14 .

\section{Results}

\section{Selection of study participants}

Out of 2307 households with PLW identified in 216 villages, 1296 households were visited and 1238 mothers in the two districts completed the survey; eight mothers were excluded, as they were not pregnant or lactating within 6 months after delivery. As a result, 1230 mothers, 589 pregnant women and 641 lactating women, were included in the analysis.

\section{General characteristics of study population}

The mean household size was 4.9 (SD $2 \cdot 1$ ) and $78 \%$ of all households had access to protected drinking-water sources (Table 1). The mean age of PLW was 25.9 (SD 6.5) years; $67 \%$ of them could read and write. A majority (93\%) of mothers were currently married. The primary occupation and main source of income was agricultural farming (83\%). The mothers' mean mid-upper arm circumference was $26 \cdot 2$ (SD $2 \cdot 6) \mathrm{cm}$.

\section{Description of housebold food insecurity and dietary diversity practices}

Among pregnant women ( $n$ 589), $14.3 \%$ were food secure; $19.0 \%$ experienced mild food insecurity, $23.9 \%$ reported moderate food insecurity and $42 \cdot 8 \%$ were

Table 1 Household- and individual-level characteristics of study pregnant and lactating women ( $n$ 1230), rural Malawi, JanuaryMarch 2014

\begin{tabular}{|c|c|c|}
\hline & Mean or \% & SD \\
\hline \multicolumn{3}{|l|}{ Household level } \\
\hline \multicolumn{3}{|l|}{ District location (\%) } \\
\hline District 1 & 51.9 & - \\
\hline District 2 & $48 \cdot 1$ & - \\
\hline Household size, mean and SD & 4.9 & $2 \cdot 1$ \\
\hline \multicolumn{3}{|l|}{ Access to drinking-water (\%) } \\
\hline Unprotected drinking-water source & $22 \cdot 2$ & - \\
\hline Protected drinking-water source & $77 \cdot 8$ & - \\
\hline \multicolumn{3}{|l|}{ Individual level } \\
\hline Age (years), mean and SD & $25 \cdot 9$ & 6.5 \\
\hline Education (years), mean and SD & $5 \cdot 3$ & 3.4 \\
\hline Literacy (\%) & $67 \cdot 0$ & - \\
\hline \multicolumn{3}{|l|}{ Marital status (\%) } \\
\hline Currently married & 92.9 & - \\
\hline Widowed/separated & $7 \cdot 1$ & - \\
\hline \multicolumn{3}{|l|}{ Occupation (\%) } \\
\hline Housewife/student & 8.5 & - \\
\hline Wage employment & $8 \cdot 7$ & - \\
\hline Farming/agriculture & $82 \cdot 8$ & - \\
\hline MUAC $(\mathrm{cm})$, mean and SD & $26 \cdot 2$ & 2.6 \\
\hline
\end{tabular}

MUAC, mid-upper arm circumference. 
categorized as severely food insecure. The distribution of household food insecurity among lactating women ( $n$ 641) was comparable to that of pregnant women $(P=0.74$; Table 2).

Among pregnant women, the mean DDS was low at $3 \cdot 1$ (SD $1 \cdot 1$ ) and $32.4 \%$ of them met the MDD classification (consumption of three or more food groups in the past $24 \mathrm{~h}$; Table 2). Among lactating women, the DDS was 3.0 (sD 1.0 ) and $28.1 \%$ consumed three or more foods groups in the last $24 \mathrm{~h}$.

Almost all women consumed grains (98.5\%), most of them had DGLV (88.6\%) and some of them had legumes $(28.7 \%)$ in the $24 \mathrm{~h}$ preceding the survey (Fig. 1). Few of them consumed nutrient-rich food groups such as meat/

Table 2 Household food insecurity and dietary diversity of study pregnant and lactating women ( $n$ 1230), rural Malawi, JanuaryMarch 2014

\begin{tabular}{lccc}
\hline & $\begin{array}{c}\text { Pregnant } \\
\text { women } \\
(n \text { 589) }\end{array}$ & $\begin{array}{c}\text { Lactating } \\
\text { women } \\
(n \text { 641) }\end{array}$ & $P^{*}$ \\
\hline Independent variable & & & \\
$\quad$ Household food insecurity (\%) & & & \\
$\quad$ Food secure & 14.3 & 13.9 & 0.74 \\
$\quad$ Mildly insecure & 19.0 & 17.5 & \\
$\quad$ Moderately insecure & 23.9 & 26.5 & \\
$\quad$ Severely insecure & 42.8 & 42.1 & \\
Dependent variables & & & \\
DDS, mean† & 3.1 & 3.0 & 0.14 \\
$\quad$ SD & 1.1 & 1.0 & \\
MDD ( $\geq 3$ food groups) (\%) & 32.4 & 28.1 & 0.10 \\
\hline
\end{tabular}

DDS, dietary diversity score; MDD, minimum dietary diversity. ${ }^{*} P$ values were calculated by $x^{2}$ test.

tDDS measures food consumption in the $24 \mathrm{~h}$ preceding the survey and is calculated using the nine following food groups: (i) starchy staples (cereals and white roots and tubers); (ii) dark green leafy vegetables; (iii) vitamin A-rich fruits and vegetables; (iv) organ meat; (v) flesh meat, fish and insects; (vi) eggs; (vii) legumes, nuts and seeds; and (ix) milk and dairy products. fish and eggs. Consumption of other types of fruits and vegetables (OFV) than vitamin A-rich ones (24.8 v. 18.4\%; $P<0 \cdot 01)$ and meat/poultry/fish (24.8 v. 19.8\%; $P=0.04)$ was low but higher among pregnant women than lactating women.

\section{Association between bousebold food insecurity and dietary diversity}

\section{Dietary diversity}

Compared with food-secure pregnant women, those reporting severe food insecurity had a decrease of 0.36 in DDS $(P=0 \cdot 02)$. However, those reporting mild or moderate food insecurity did not show a significant difference in DDS, compared with the food-secure pregnant group (Table 3). Compared with food-secure pregnant women, those reporting severe food insecurity reported 2.96 times higher risk of not achieving MDD in the unadjusted model $(P<0.01)$, but the OR was attenuated to $1.83(P=0.07)$ when fully adjusted.

Among lactating women, mild, moderate and severe food insecurity was associated with DDS (0.36, 0.44 and 0.62 lower scores, respectively), compared with the foodsecure group (Table 3; all $P<0.05$ ). Lactating women who reported moderate or severe food insecurity were at 1.95 and 2.82 higher risk of not achieving MDD, respectively, compared with the food-secure group (all $P<0 \cdot 05$ ). However, mild food insecurity was not significantly related to DDS or MDD.

\section{Food group consumption}

Compared with food-secure pregnant women, severe food insecurity was associated with threefold $(\mathrm{OR}=3 \cdot 19$; $P<0.01)$ greater risk of not having meat/fish in the past $24 \mathrm{~h}$ (Table 4). Also, the risk of not consuming eggs

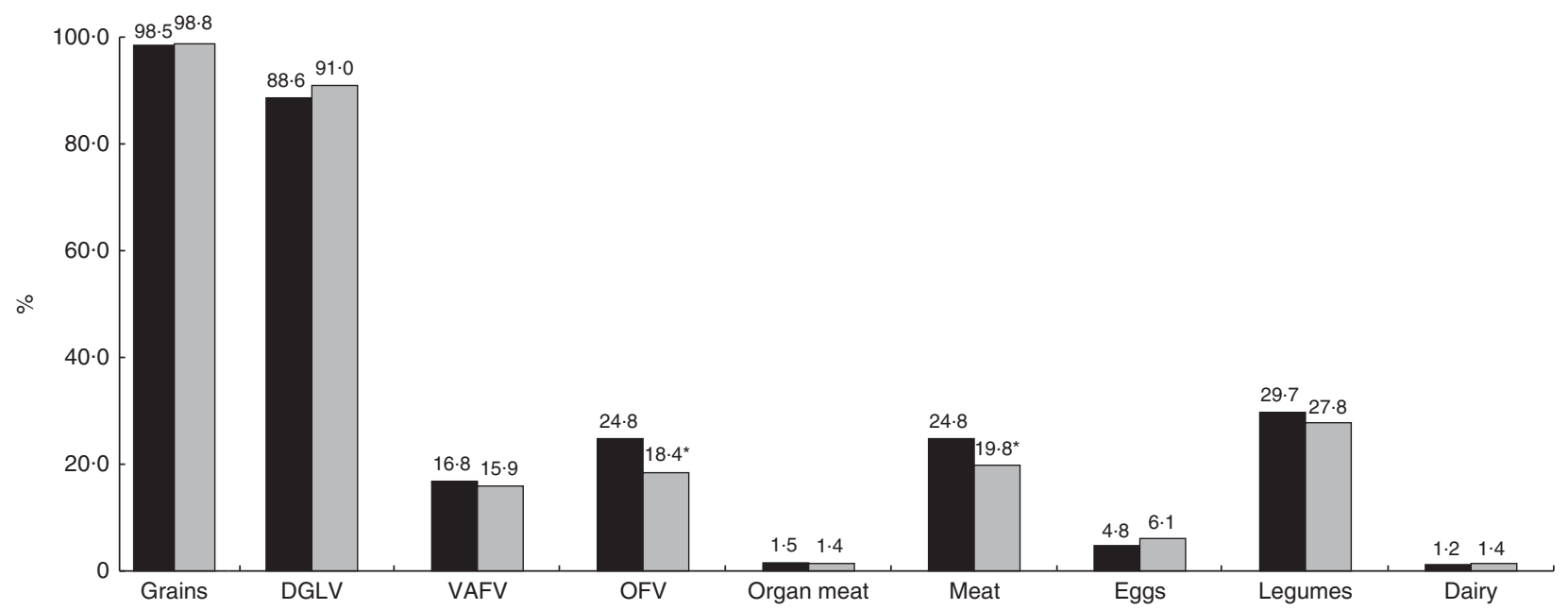

Fig. 1 Food group consumption in the past $24 \mathrm{~h}$ among study pregnant women $(n 589 ; \square)$ and lactating women $(n 641 ; \square)$, rural Malawi, January-March 2014. ${ }^{*} P<0.05$ (DGLV, dark green leafy vegetables; VAFV, vitamin A-rich fruits and vegetables; OFV, other fruits and vegetables) 
Table 3 Association between household food insecurity and dietary diversity among study pregnant and lactating women ( $n$ 1230), rural Malawi, January-March 2014

\begin{tabular}{|c|c|c|c|c|c|c|c|c|c|c|c|c|}
\hline & \multicolumn{3}{|c|}{ Secure } & \multicolumn{3}{|c|}{ Mildly insecure } & \multicolumn{3}{|c|}{ Moderately insecure } & \multicolumn{3}{|c|}{ Severely insecure } \\
\hline & $\begin{array}{l}\beta \text { or } \\
\text { OR }\end{array}$ & $95 \% \mathrm{Cl}$ & $P$ & $\begin{array}{l}\beta \text { or } \\
\text { OR }\end{array}$ & $95 \% \mathrm{Cl}$ & $P$ & $\begin{array}{l}\beta \text { or } \\
\text { OR }\end{array}$ & $95 \% \mathrm{Cl}$ & $P$ & $\begin{array}{l}\beta \text { or } \\
\text { OR }\end{array}$ & $95 \% \mathrm{Cl}$ & $P$ \\
\hline \multicolumn{13}{|c|}{ Pregnant women $(n$ 589) } \\
\hline Unadjusted & - & - & - & -0.21 & $-0.54,0.11$ & 0.20 & -0.22 & $-0.51,0.07$ & 0.14 & -0.69 & $-0.96,-0.43$ & $<0.01$ \\
\hline Adjusted $\dagger$ & - & - & - & -0.10 & $-0.44,0.24$ & 0.57 & -0.05 & $-0.37,0.26$ & 0.74 & -0.36 & $-0.68,-0.05$ & 0.02 \\
\hline \multicolumn{13}{|c|}{$\begin{array}{l}\text { Not meeting MDD } \\
\text { ( }<3 \text { food groups) }\end{array}$} \\
\hline Unadjusted & 1.00 & Ref. & - & $1 \cdot 12$ & $0.53,2.34$ & 0.77 & 1.50 & $0.79,2.85$ & 0.22 & 2.96 & $1 \cdot 69,5 \cdot 17$ & $<0.01$ \\
\hline Adjusted & 1.00 & Ref. & - & 0.94 & $0.44,2.02$ & 0.88 & $1 \cdot 18$ & $0.59,2.37$ & 0.65 & 1.83 & $0.96,3.49$ & 0.07 \\
\hline \multirow{2}{*}{\multicolumn{13}{|c|}{ Lactating women ( $n$ 641) }} \\
\hline & & & & & & & & & & & & \\
\hline Unadjusted & - & - & - & -0.37 & $-0.67,-0.08$ & 0.01 & -0.53 & $-0.82,-0.23$ & $<0.01$ & -0.88 & $-1.17,-0.60$ & $<0.01$ \\
\hline Adjusted & - & - & - & -0.36 & $-0.64,-0.08$ & 0.01 & -0.44 & $-0.72,-0.16$ & $<0.01$ & -0.62 & $-0.89,-0.35$ & $<0.01$ \\
\hline \multicolumn{13}{|c|}{$\begin{array}{l}\text { Not meeting MDD } \\
\text { (<3 food groups) }\end{array}$} \\
\hline Unadjusted & 1.00 & Ref. & - & 1.48 & $0.77,2.85$ & 0.24 & $2 \cdot 14$ & $1.16,3.93$ & 0.02 & $3 \cdot 87$ & $2 \cdot 15,6.99$ & $<0.01$ \\
\hline Adjusted & 1.00 & Ref. & - & 1.47 & $0.76,2.83$ & 0.25 & 1.95 & $1.06,3.59$ & 0.03 & $2 \cdot 82$ & $1.53,5.22$ & $<0.01$ \\
\hline
\end{tabular}

DDS, dietary diversity score; MDD, minimum dietary diversity; Ref., reference category.

*DDS measures food consumption in the $24 \mathrm{~h}$ preceding the survey and is calculated using the nine following food groups: (i) starchy staples (cereals and white roots and tubers); (ii) dark green leafy vegetables; (iii) vitamin A-rich fruits and vegetables; (iv) organ meat; (v) flesh meat, fish and insects; (vi) eggs; (vii) legumes, nuts and seeds; and (ix) milk and dairy products.

†All linear or logistic regressions were adjusted for district location, household wealth status, drinking-water source, household size, age, literacy, years of education, occupation, marital status and mid-upper arm circumference. Study design effect was accounted for in all models.

increased by almost four times $(\mathrm{OR}=3.77 ; \quad P=0.04)$ among pregnant women reporting moderate food insecurity. However, the risk of not having other food groups did not differ with severity of food security among pregnant women (see online supplementary material, Supplemental Table 2).

The risk of not consuming meat increased with strength of household food insecurity among lactating mothers (Table 4). The risk increased by 1.75, 2.66 and 5.33 times among the mildly, moderately and severely food-insecure group, respectively, compared with the food-secure group (all $P<0.05$ ). The risk of not consuming eggs increased by $2.81,3.75$ and 3.47 times among mildly, moderately and severely food-insecure women, respectively, compared with the food-secure group (all $P<0.05$ ). The risk of not having legumes increased among only the severely food-insecure group ( $\mathrm{OR}=1.94 ; P=0.04)$ but not in the mildly or moderately food-insecure group. The likelihood of not consuming other food groups did not differ with varying household food insecurity among lactating women (see online supplementary material, Supplemental Table 3).

\section{Discussion}

Sustainable Development Goal 2 aims to eradicate hunger and all types of malnutrition by $2030^{(31)}$. To succeed in this goal, ensuring proper nutrition by securing food access and availability to all vulnerable populations, especially PLW, is essential. Our study showed that household food insecurity was associated with low dietary diversity among both pregnant women and lactating women in rural Malawi. While pregnant women's diet was associated with only severe food insecurity, lactating women were likely to be affected even by mild household food insecurity. The risk of not consuming animal-source foods such as meat/poultry/fish or eggs was high among food-insecure pregnant women and even higher among food-insecure lactating women.

The traditional rural Malawian diet consists of nsima (a maize-based dish), boiled vegetables and relish. Grainbased diets, with low consumption of animal protein and poor intakes of $\mathrm{Zn}$ and $\mathrm{Fe}$, were reported among $\mathrm{PLW}^{(32-34)}$. Our study also highlighted the monotonous diet of women in this setting, which is primarily based on grains and DGLV, and is lacking in micronutrients and animal-source protein from animal fleshed foods, dairy products and eggs.

Household food insecurity experience typically begins with worry about food shortages. As this situation worsens, compromises to dietary quality and variety increase. Limiting the quantity of food consumed occurs only under severe circumstances ${ }^{(35,36)}$. In such situations, intrahousehold food allocation is affected by severe or unexpected food insecurity and women's diet is often the first to be compromised. Women use their decision-making power or control over resources to maximize family members', rather than their own, nutritional outcomes ${ }^{(37)}$.

In Malawi, food security varies by season. In the dry season (March to August), food availability is usually high, while food shortages are more common in the wet, lean season (September to February) ${ }^{(38)}$. Given the timing of data collection in the present study (January to March), the 
Table 4 Association between household food insecurity and food group consumption among study pregnant and lactating women ( $n 1230)$, rural Malawi, January-March 2014

\begin{tabular}{|c|c|c|c|c|c|c|c|c|c|c|c|c|}
\hline & \multicolumn{3}{|c|}{ Secure } & \multicolumn{3}{|c|}{ Mildly insecure } & \multicolumn{3}{|c|}{ Moderately insecure } & \multicolumn{3}{|c|}{ Severely insecure } \\
\hline & OR & $95 \% \mathrm{Cl}$ & $P$ & OR & $95 \% \mathrm{Cl}$ & $P$ & OR & $95 \% \mathrm{Cl}$ & $P$ & OR & $95 \% \mathrm{Cl}$ & $P$ \\
\hline \multicolumn{13}{|c|}{ Pregnant women ( $n$ 589) } \\
\hline \multicolumn{13}{|l|}{ No meat/fish } \\
\hline Unadjusted & 1.00 & Ref. & - & 1.45 & $0.76,2.75$ & 0.26 & 1.74 & $1.00,3.05$ & 0.05 & 4.74 & $2.81,8.00$ & $<0.01$ \\
\hline Adjusted ${ }^{*}$ & 1.00 & Ref. & - & 1.25 & $0.64,2.47$ & 0.51 & 1.45 & $0.77,2 \cdot 71$ & 0.25 & $3 \cdot 19$ & $1.68,6.03$ & $<0.01$ \\
\hline \multicolumn{13}{|l|}{ No eggs } \\
\hline Unadjusted & 1.00 & Ref. & - & 3.22 & $1 \cdot 14,9 \cdot 10$ & 0.03 & $5 \cdot 16$ & $1.58,16 \cdot 8$ & $<0.01$ & 4.60 & $1.80,11.7$ & $<0.01$ \\
\hline Adjusted & 1.00 & Ref. & - & 2.57 & $0.84,7.85$ & 0.10 & 3.77 & $1.04,13.7$ & 0.04 & 2.57 & $0.86,7.62$ & 0.09 \\
\hline \multicolumn{13}{|c|}{ Lactating women ( $n$ 641) } \\
\hline \multicolumn{13}{|c|}{ No meat/fish } \\
\hline Unadjusted & 1.00 & Ref. & - & 1.78 & $1.06,2.98$ & 0.03 & 2.85 & $1.66,4.88$ & $<0.01$ & $7 \cdot 29$ & $3.96,13.4$ & $<0.01$ \\
\hline Adjusted & 1.00 & Ref. & - & 1.75 & $1.01,3.03$ & 0.05 & $2 \cdot 66$ & $1.47,4.82$ & $<0.01$ & $5 \cdot 33$ & $2.63,10 \cdot 8$ & $<0.01$ \\
\hline \multicolumn{13}{|l|}{ No eggs } \\
\hline Unadjusted & 1.00 & Ref. & - & 3.29 & $1.38,7 \cdot 86$ & $<0.01$ & $4 \cdot 44$ & $1.83,10.7$ & $<0.01$ & $7 \cdot 18$ & $2.98,17.3$ & $<0.01$ \\
\hline Adjusted & 1.00 & Ref. & - & $2 \cdot 81$ & $1 \cdot 09,7 \cdot 25$ & 0.03 & 3.75 & $1.40,10.0$ & 0.01 & 3.47 & $1 \cdot 19,10 \cdot 1$ & 0.02 \\
\hline \multicolumn{13}{|l|}{ No legumes } \\
\hline Unadjusted & 1.00 & Ref. & - & $1 \cdot 31$ & $0.75,2.30$ & 0.34 & 1.51 & $0.88,2.60$ & 0.14 & 2.53 & $1.42,4.52$ & $<0.01$ \\
\hline Adjusted & 1.00 & Ref. & - & 1.42 & $0.75,2.68$ & 0.28 & 1.38 & $0.77,2.48$ & 0.28 & 1.94 & $1.04,3.62$ & 0.04 \\
\hline
\end{tabular}

Ref., reference category.

*All logistic regressions were adjusted for district location, household wealth status, drinking-water source, household size, age, literacy, years of education, occupation, marital status and mid-upper arm circumference. Study design effect was accounted for in all models.

percentage of women experiencing moderate or severe food insecurity (68.0\%) would likely be worse than during other times of the year.

The increased risk of not having meat/fish/poultry or eggs implies that the nature of food insecurity in this setting compromises the quality of some diets, given that they are relatively more expensive and nutrient-rich than other foods. Even severe food insecurity among PLW was not associated with reduced consumption of staple foods such as grains and some vegetables (see online supplementary material, Supplemental Tables 2 and 3), which tells us that perhaps staple foods are available to households regardless of whether the household has a sufficient food supply or not. This pattern is consistent with the recent increased availability of grains resulting from strengthened government subsidy programmes that have focused on maize production $^{(21)}$.

The food groups that were associated with severe food insecurity were not always associated with less severe food-insecure conditions (i.e. mildly or moderately). For example, consumption of legumes was associated with moderate food insecurity, but the association was attenuated by more severe conditions among lactating women.

Our study emphasized that household food insecurity and low dietary diversity are problems not only among pregnant but also lactating women in rural Malawi. In the study area, lactating women might be at a higher risk for poor dietary diversity than pregnant women if food insecurity deteriorates. While pregnant women, unless they were in a severely constrained condition, were able to meet a minimum level of dietary diversity, lactating women were at risk of not meeting the MDD even in less severe conditions. Given that the condition of HFIAS was not different between the pregnant women and lactating women $\left(P=0 \cdot 74\right.$, tested by $\chi^{2}$ test; Table 3$)$, the reasons for varying ability to achieve the lowest dietary diversity between these groups should be examined further. However, we hypothesize that pregnant women may be protected from worsened food insecurity given their upcoming birth, while lactating mothers may be treated as equal to non-lactating women despite their increased nutritional needs to support lactation. If so, continued nutrition education at antenatal and postnatal health visits should not underestimate the importance of a promoting a high-quality diet during lactation.

A handful of studies have examined the association between dietary diversity and household food security or socio-economic status among women, as a proxy indicator for food insecurity in resource-poor settings. In a crosssectional study of 15899 women of reproductive age in Nepal, higher socio-economic status was associated with higher consumption of most food groups except for cereal-based foods, fats and edible oils, and flesh food groups $^{(10)}$. Another cross-sectional study of 508 pregnant women in south-western Bangladesh revealed that an economically poorer condition was associated with lower consumption of dairy and eggs ${ }^{(15)}$. In a study of $161 \mathrm{HIV}-$ negative and $514 \mathrm{HIV}$-infected Rwandan women, low income was related to food insufficiency and consuming three or fewer food groups, but low BMI $\left(\leq 18.5 \mathrm{~kg} / \mathrm{m}^{2}\right)$ was not related to food insufficiency and dietary diversity ${ }^{(3)}$. Only one study examined the association between household food security, as a perception-based quantitative measure, and dietary diversity in a longitudinal cohort following women from pregnancy through lactation in 
rural Bangladesh ${ }^{(14)}$. In that study, higher food insecurity in households was associated with decreased intakes of animal flesh foods, dairy products, eggs and legumes among women. Our study findings are consistent with such findings and contribute to our general understanding of the association between food insecurity and women's diets in the rural sub-Saharan African setting.

A strength of the present study is the large sample size of PLW. Unlike most of studies that did not distinguish the condition of pregnancy and lactation or targeted only one group, our effort adds detail and richness by comparing the patterns of association between food insecurity and diet profiles of two vulnerable population groups in this setting.

There are a few limitations to our study that deserve mention here. First, our study was not able to adjust for the period of pregnancy and lactation. Last menstruation period was assessed but was not used in our analysis due to limited and unreliable recall by mothers. Instead, we assumed most of the pregnant women interviewed were in their second or third trimester, given the known cultural practice in this context of not disclosing a pregnancy until it is visible ${ }^{(39)}$. Second, given our use of cross-sectional data, we are unable to infer the causal relationship between household food insecurity and dietary diversity. Third, HFIAS is a household-, rather than an individual-level indicator. In addition, some food insecurity domains such as food safety are not assessed. Further, over-reporting for experience of food insecurity may have occurred among some respondents with the expectation of receiving more support in the context of an emergency ${ }^{(25)}$. Fourth, mothers' diet in the preceding $24 \mathrm{~h}$ may not be representative of usual consumption patterns. Most mothers were assessed during weekdays, but a few were visited on Saturday. Moreover, the timing during which the study was conducted corresponded with the rainy season, during which dietary patterns differ from those seen in the dry season. Nevertheless, we assumed that the monotonous diet seen in this setting is unlikely to change significantly from one season to another. Lastly, the categorization of foods collected for the study did not allow for the calculation of ten food groups that are required to calculate the Minimum Dietary Diversity for Women (MDD-W) indicator $^{(40)}$. Particularly, out of ten groups that constitute the MDD-W, lentils, nuts/seeds, other fruits and other vegetables were not asked separately in the questionnaire. Also, organ meat was counted as an individual food group in our study, but not an individual food group in MDD-W.

These research findings demonstrate possible evidence of poor dietary diversity attributed to food insecurity, which is experienced by both pregnant women and lactating women in rural Malawi. The findings underline the need to strengthen strategies to promote food security among both pregnant women and lactating women in low-resource settings. For example, food-based activities or conditional cash transfer programmes that were integrated into existing antenatal and postnatal care service improved food security ${ }^{(41,42)}$. Women's empowerment to increase their control of household financial resources may improve access and utilization of quality foods during pregnancy and lactation ${ }^{(43)}$. Also, given the lower consumption of $\mathrm{Fe}$ - and protein-rich foods observed among food-insecure PLW in this setting, we could encourage programme practitioners to strengthen protein and micronutrient supplementation efforts or provide food vouchers to these sub-populations.

\section{Conclusion}

In conclusion, household food insecurity in rural Malawi was associated with poor dietary practices among PLW. Programme practitioners and policy makers may consider strategies to enhance food security during and after pregnancy when designing nutrition programmes aiming to increase the dietary diversity of this population.

\section{Acknowledgements}

Acknowledgements: The authors thank all study participants in the study area, Malawi. They acknowledge the World Food Programme and World Vision Malawi for their guidance and implementation support. The authors appreciate Wadonda Consult Ltd for field data collection and data management. They also thank Audrey Buckland, Johns Hopkins Bloomberg School of Public Health, USA, for the manuscript review. Financial support: This study was funded by Children's Investment Fund Foundation (CIFF), UK. The funding agency had no role in the design of the study, data collection and analysis, or presentation of the results. Conflict of interest: No authors had conflict of interest related to the study. Authorship: Y.K. and K.M.H. designed the present research; P.C., R.K. and K.P.W. designed a parental trial; A.B.M. and J.P. supervised data collection in Malawi; L.S.F.W. and M.M. managed the data set; Y.K. analysed the data and prepared the manuscript draft; K.M.H., J.R.-B., R.O. and P.C. contributed substantially to interpretation of results; all authors contributed to the writing of the manuscript; Y.K. had primary responsibility for the final content of the manuscript; all authors read and approved the final manuscript. Ethics of buman subject participation: This study was conducted according to the guidelines laid down in the Declaration of Helsinki and all procedures involving human subjects were approved by the Institutional Review Board at Johns Hopkins School of Public Health, USA and the College of Medicine Research and Ethics Committee (COMREC), University of Malawi, Blantyre, Malawi. The trial is registered to ClinicalTrials.gov (NCT02985359). All subjects 
were orally consented and informed about the study purpose and scope of assessment.

\section{Supplementary material}

To view supplementary material for this article, please visit https://doi.org/10.1017/S1368980018002719

\section{References}

1. Haileslassie K, Mulugeta A \& Girma M (2013) Feeding practices, nutritional status and associated factors of lactating women in Samre Woreda, South Eastern Zone of Tigray, Ethiopia. Nutr J 12, 28.

2. Perumal N, Cole DC, Ouedraogo HZ et al. (2013) Health and nutrition knowledge, attitudes and practices of pregnant women attending and not-attending ANC clinics in western Kenya: a cross-sectional analysis. BMC Pregnancy Childbirth 13, 146.

3. Sirotin N, Hoover D, Segal-Isaacson CJ et al. (2012) Structural determinants of food insufficiency, low dietary diversity and BMI: a cross-sectional study of HIV-infected and HIV-negative Rwandan women. BMJ Open 2, e000714.

4. Lee SE, Talegawkar SA, Merialdi M et al. (2013) Dietary intakes of women during pregnancy in low- and middleincome countries. Public Health Nutr 16, 1340-1353.

5. Black RE, Victora CG, Walker SP et al. (2013) Maternal and child undernutrition and overweight in low-income and middle-income countries. Lancet 382, 427-451.

6. Innis SM (2014) Impact of maternal diet on human milk composition and neurological development of infants. $\mathrm{AmJ}$ Clin Nutr 99, issue 3, 734S-741S.

7. Ballard O \& Morrow AL (2013) Human milk composition: nutrients and bioactive factors. Pediatr Clin North Am 60, 49-74.

8. Lonnerdal B (1986) Effects of maternal dietary intake on human milk composition. J Nutr 16, 499-513.

9. UNICEF (1997) The State of the World's Children 1997. New York: UNICEF.

10. Campbell RK, Talegawkar SA, Christian P et al. (2014) Seasonal dietary intakes and socioeconomic status among women in the Terai of Nepal. J Health Popul Nutr 32, 198-216.

11. Harris-Fry H, Azad K, Kuddus A et al. (2015) Socioeconomic determinants of household food security and women's dietary diversity in rural Bangladesh: a crosssectional study. J Health Popul Nutr 33, 2.

12. Islam MZ, Akhtaruzzaman M \& Lamberg-Allardt C (2004) Nutritional status of women in Bangladesh: comparison of energy intake and nutritional status of a low income rural group with a high income urban group. Asia Pac J Clin Nutr 13, 61-68.

13. Nguyen PH, Strizich G, Lowe A et al. (2013) Food consumption patterns and associated factors among Vietnamese women of reproductive age. Nutr J 12, 126.

14. Na M, Mehra S, Christian P et al. (2016) Maternal dietary diversity decreases with household food insecurity in rural Bangladesh: a longitudinal analysis. J Nutr 146, 2109-2116.

15. Shamim AA, Mashreky SR, Ferdous T et al. (2016) Pregnant women diet quality and its sociodemographic determinants in southwestern Bangladesh. Food Nutr Bull 37, $14-26$.

16. Stevens B, Watt K, Brimbecombe J et al. (2017) The role of seasonality on the diet and household food security of pregnant women living in rural Bangladesh: a crosssectional study. Public Health Nutr 20, 121-129.
17. National Statistical Office (Malawi) \& ICF (2017) Malawi Demographic and Health Survey 2015-16. Zomba, Malawi and Rockville, MD: NSO and ICF.

18. Huddle JM, Gibson RS \& Cullinan TR (1999) The impact of malarial infection and diet on the anaemia status of rural pregnant Malawian women. Eur J Clin Nutr 53, 792-801.

19. Munasinghe $S$ \& van den Broek N (2006) Anaemia in pregnancy in Malawi - a review. Malawi Med J 18, 160-174.

20. Harrigan J (2008) Food insecurity, poverty and the Malawian Starter Pack: fresh start or false start? Food Policy $\mathbf{3}$, 237-249.

21. Verduzco-Gallo I, Olivier Ecker O \& Pauw K (2014) Changes in Food and Nutrition Security in Malawi: Analysis of Recent Survey Evidence. MaSSP Working Paper no 6. Washington, DC: International Food Policy Research Institute.

22. Malawi National Statistical Office (2015) Malawi Statistical Yearbook 2015. Zomba, Malawi: NSO.

23. Malawi National Statistical Office (2012) Integrated Housebold Survey (IHS3) 2010-2011: Household Socio-Economic Characteristics Report. Zomba, Malawi: NSO.

24. World Food Programme (2015) How WFP Supported the Scaling Up Nutrition (SUN) Roll-Out in Malawi: A Case Study on Stunting Prevention Programming at Scale. Rome: WFP.

25. Food and Nutrition Technical Assistance Project (2007) Household Food Insecurity Access Scale (HFIAS) for Measurement of Food Access: Indicator Guide. Washington, DC: FANTA, Academy for Educational Development.

26. Knueppel D, Demment M \& Kaiser L (2010) Validation of the Household Food Insecurity Access Scale in rural Tanzania. Public Health Nutr 13, 360-367.

27. Gebreyesus S, Lunde T, Mariam D et al. (2015) Is the adapted Household Food Insecurity Access Scale (HFIAS) developed internationally to measure food insecurity valid in urban and rural households of Ethiopia?. BMC Nutr 1, 2.

28. Desiere S, D'Haese M \& Niragira S (2015) Assessing the cross-sectional and inter-temporal validity of the Household Food Insecurity Access Scale (HFIAS) in Burundi. Public Health Nutr 18, 2775-2785.

29. Food and Agriculture Organization of the United Nations (2011) Guidelines for Measuring Housebold and Individual Dietary Diversity. Rome: FAO.

30. Vyas S \& Kumaranayake L (2016) Constructing socioeconomic status indices: how to use principal components analysis. Health Policy Plan 21, 459-468.

31. United Nations (2015) Sustainable Development Goals: Knowledge Platform. https://sustainabledevelopment.un. org/sdgs (accessed January 2017).

32. Gibson RS \& Huddle JM (1998) Suboptimal zinc status in pregnant Malawian women: its association with low intakes of poorly available zinc, frequent reproductive cycling, and malaria. Am J Clin Nutr 67, 702-709.

33. Siyame EW, Hurst R, Wawer AA et al. (2013) A high prevalence of zinc- but not iron-deficiency among women in rural Malawi: a cross-sectional study. Int J Vitam Nutr Res 83, 176-187.

34. Ramlal RT, Tembo M, Soko A et al. (2013) Patterns of body composition among HIV-infected, pregnant Malawians and the effects of famine season. Matern Child Health J 17, 265-273.

35. Radimer KL, Olson CM \& Campbell CC (1990) Development of indicators to assess hunger. J Nutr 120, Suppl. 11, 1544-1548.

36. Radimer KL, Olson CM, Greene JC et al. (1992) Understanding hunger and developing indicators to assess it in women and children. J Nutr Educ 24, Suppl. 1, S36-S44.

37. Harris-Fry H, Shrestha N, Costello A et al. (2017) Determinants of intra-household food allocation between adults in South Asia - a systematic review. Int J Equity Health 16, 107.

38. Chikhungu LC \& Madise NJ (2014) Seasonal variation of child under nutrition in Malawi: is seasonal food availability 
an important factor? Findings from a national level crosssectional study. BMC Public Health 14, 1146.

39. Roberts J, Hopp Marshak H, Sealy DA et al. (2017) The role of cultural beliefs in accessing antenatal care in Malawi: a qualitative study. Public Health Nurs 34, 42-49.

40. Food and Agriculture Organization of the United Nations \& FHI 360 (2016) Minimum Dietary Diversity for Women: A Guide for Measurement. Rome: FAO.

41. Nguyen PH, Kim SS, Sanghvi T et al. (2017) Integrating nutrition interventions into an existing maternal, neonatal, and child health program increased maternal dietary diversity, micronutrient intake, and exclusive breastfeeding practices in Bangladesh: results of a cluster-randomized program evaluation. J Nutr 147, 2326-2337.

42. Raghunathan K, Chakrabarti S, Avula R et al. (2017) Can conditional cash transfers improve the uptake of nutrition interventions and household food security? Evidence from Odisha's Mamata scheme. PLoS One 12, e0188952.

43. van den Bold M, Dillon A, Olney D et al. (2015) Can integrated agriculture-nutrition programmes change gender norms on land and asset ownership? Evidence from Burkina Faso. J Dev Stud 51, 1155-1174. 